\title{
Concurrent Model Synchronisation with Multiple Objectives
}

\author{
Nils Weidmann \\ Department of Computer Science \\ Paderborn University, Germany \\ Paderborn, Germany \\ nils.weidmann@upb.de
}

\author{
Gregor Engels \\ Department of Computer Science \\ Paderborn University, Germany \\ Paderborn, Germany \\ engels@upb.de
}

\begin{abstract}
Concurrent model synchronisation, i.e. the (bidirectional) propagation of updates between two models, is an important problem in the area of model-driven engineering (MDE). Compared to other consistency management tasks, synchronising concurrent updates is especially challenging as they can be conflicting, such that restoring a consistent state is not possible when all updates must be considered. Recent approaches create a search space of possible solutions and determine the optimum solution via exact methods, such as integer linear programming (ILP), via a configurable, scalarised objective function that takes conflicting goals into account. However, the determination of suitable configuration parameters and runtime efficiency improvements are still an open issue, which is commonly addressed by using heuristics instead of exact methods. We investigate on whether it is beneficial to apply heuristics to solve concurrent model synchronisation problems. First, a multiobjective evolutionary algorithm is used for small instances for which all pareto-optimal solutions can be presented to a user to select the best one. Second, for larger models, we propose a method to determine suitable weightings for aggregating all objectives into a single function. Finally, these insights are used to recommend a strategy for determining solutions of satisfying quality within an acceptable amount of time.
\end{abstract}

\section{CCS CONCEPTS}

- Software and its engineering $\rightarrow$ Model-driven software engineering; Search-based software engineering.

\section{KEYWORDS}

Model synchronisation, search-based software engineering, multiobjective optimization

\section{ACM Reference Format:}

Nils Weidmann and Gregor Engels. 2021. Concurrent Model Synchronisation with Multiple Objectives. In 2021 Genetic and Evolutionary Computation Conference (GECCO '21), fuly 10-14, 2021, Lille, France. ACM, New York, NY, USA, 9 pages. https://doi.org/10.1145/3449639.3459283

Permission to make digital or hard copies of all or part of this work for personal or classroom use is granted without fee provided that copies are not made or distributed for profit or commercial advantage and that copies bear this notice and the full citation on the first page. Copyrights for components of this work owned by others than ACM must be honored. Abstracting with credit is permitted. To copy otherwise, or republish, to post on servers or to redistribute to lists, requires prior specific permission and/or a fee. Request permissions from permissions@acm.org.

GECCO '21, July 10-14, 2021, Lille, France

(C) 2021 Association for Computing Machinery.

ACM ISBN 978-1-4503-8350-9/21/07.

https://doi.org/10.1145/3449639.3459283

\section{INTRODUCTION}

To cope with nowadays complexity of software systems, ModelDriven Engineering (MDE) considers models as primary artefacts in the development process. Maintaining consistency between the involved models becomes important in collaborative settings, in which multiple (teams of) domain experts work on different, but semantically interrelated models. Thereby, the synchronisation of models after concurrent updates, in short concurrent model synchronisation, is an especially challenging task. Updates, e.g., refactorings on one model can be in conflict with updates on the other, such that it is hard to find a satisfactory solution for all involved stakeholders. The problem is similar to model versioning, as the goal is to reach a consistent state after independent changes by different users. In concurrent synchronisation scenarios, in contrast, multiple models are to be kept consistent, which have different metamodels and therefore require more advanced resolution strategies.

There are several possible policies to resolve these conflicts and eventually restore consistency. Some recent approaches detect conflicts explicitly and resolve them according to a local decision strategy [15]. In other works, an exhaustive search over all possible solutions is conducted [28], which easily gets intractable to solve due to a combinatorial explosion of system states. An implementation for synchronising concurrent updates based on Triple Graph Grammars (TGGs) and Integer Linear Programming (ILP) techniques was recently presented [32]. As a major criticism, the authors pointed out that ILP as an exact method does not scale well for larger model sizes. It might pay off using a heuristic that only finds a near-optimal solution but shows a much better runtime performance. Furthermore, the objective function is composed of four parts which balance conflicting goals, such as preserving deletions and creations, keeping unchanged model parts as they are, and reducing the amount of automated changes. The four goal functions are weighted with configuration parameters, such that the optimality of a solution heavily depends on their choice. The values were arbitrarily chosen for demonstration purposes, such that the determination of weighting parameters that lead to a satisfactory synchronisation solution was left to future work.

We propose a heuristic approach to concurrent model synchronisation that extends the work of Weidmann et al. [32] by using heuristic search algorithms that were successfully applied to model merging already $[6,14,22]$. The concurrent synchronisation problem is modelled as a multi-objective optimisation problem with four objectives, from which a set of pareto-optimal solutions is computed. It is to be expected, however, that the set of solutions will be too large even for medium-sized models to let a user choose the final solution from it. Therefore, suitable weighting parameters 
are determined in an empirical use case to form a scalarised single objective. Finally, the runtime performance and the achieved quality is compared to the baseline presented in prior work using models of different sizes.

The rest of the paper is structured as follows: After providing an overview of related work (Sect. 2), the adaption of the heuristic search algorithms for the problem domain is presented in Sect. 3. The results of applying our approach to a use case is described in Sect. 4, as well as an experimental evaluation that compares its runtime performance and result quality to an existing baseline. A summary and ideas for subsequent research are sketched in Sect. 5.

\section{RELATED WORK}

Search-based techniques have been applied in MDE for several years, whereby three main application areas can be identified. Model transformation denotes the consistent translation of entire models into another domain. The transformation is either based on rules or on examples of consistently transformed model pairs, which is denoted as Model Transformation by Example (MTBE) [18]. Model synchronisation takes existing models together with information about user edits as input and restores a consistent state by adapting both models [3]. When different versions of the same model are edited in parallel, model merging $[29,33]$ combines these changes into a new synchronised model.

Several heuristics were recently applied to address these model management problems. From the group of evolutionary algorithms, Genetic Algorithm (GA) [17] and Non-dominated Sorting Genetic Algorithm II (NSGA-II) [13] are most commonly used for singleand multi-objective optimisation, respectively. Kessentini et al. [22] proposed an approach for merging different model versions based on GA, maximising the number of edit operations to create the merged model. Similarly, Assuncao et al. [4] used GA to merge UML models with the goal of minimising the difference between the input models and the merged model. Taking the importance of edit operations into account as a second objective, Mansoor et al. [27] performed model merging using NSGA-II. When analysing the results of a model transformation, the applied steps are of particular interest. Based on a list of possible steps, which can be fine-grained (e.g., graph edits) or high-level (e.g., refactorings), a search space of application sequences can be constructed, which is usually too large for performing an exhaustive search. Both GA [5], NSGA-II [30] and its predecessor NSGA [1] were used as meta-heuristics for efficiently exploring this search space.

Local search strategies start with a (random) initial solution and iteratively improve it until some termination condition is reached. Debreceni et al. [14] use guided local search for merging different model versions, while conflicts emerge as constraint violations. Besides this, Design-Space Exploration (DSE) was used to determine optimal merging results, which was also applied to other problem domains $[12,16]$. Good runtime performance results were received on benchmark instances of 50,000 model elements. Dam et al. [10, 11] propose a local search algorithm for merging uncontroversial changes in different model versions and detect conflicts, which are subsequently presented to the user. Simulated Annealing (SA) [23] is a local search algorithm that accepts worse intermediate solutions with some probability to escape local optima. This strategy is used by Kessentini et al. [19-21] in combination with particle swarm optimisation for MTBE, showing promising results for small model instances.

Concurrent model synchronisation, to the best of our knowledge, was only conducted using exact methods, though. Kleiner et al. [24] formally specify the transformation via a third transformation model. Via constraint programming, an extension of this model triple is determined, which is considered as a correct transformation result if it conforms to its metamodel. Macedo et al. [26] find the closest consistent model based on constraint solving and a distance function for model changes. Similarly, Cicchetti et al. [8] determine sub-models with minimal edit distance to the input models which are consistent with respect to their consistency specification. In a comparative study [2], however, these implementations showed below-average performance due to the large amount of constraints which is necessary to encode graph properties. Orejas et al. [28] recently presented a TGG-based approach, which performs a global search on rule application level, which restricts the search space to a manageable size compared to purely constraint-based approaches. To determine conflicting changes on the input models and all possible solutions for resolving these conflicts, backtracking is used. An implementation is left to future work, and due to the computational effort to perform an exhaustive search for all possible rule application sequences, it is questionable whether this approach is feasible to be used in practice for larger models. Similarly, Weidmann et al. [32] optimise on rule application level by combining TGGs with ILP techniques. A performance evaluation has shown scalability problems for large models, such that the authors suggested to use (meta-)heuristics to overcome this problem.

In summary, both evolutionary algorithms and different local search strategies were successfully applied in model merging and model transformation scenarios, whereas concurrent synchronisation approaches solely rely on exact methods up to now. In a comparative study, Bill et al. [6] applied different meta-heuristics to model transformation, concluding that SA outperforms other local search algorithms for larger models. Compared to GA, SA shows better results for search spaces with a large infeasible region, whereas the opposite is the case when most solutions of the search space are valid. As the combined approach of TGGs and ILP performed comparably well for medium-sized models in prior experiments, we will continue with this idea to analyse the potential of GA, NSGA-II and SA for concurrent model synchronisation.

\section{CONCEPT}

In this section, the problem of synchronising models after concurrent updates is motivated with an illustrative example, such that the solution approach, i.e. the construction of the search space and the adaption of meta-heuristics for efficiently retrieving appropriate solution can be explained subsequently.

\subsection{Motivating Example}

To demonstrate the problem and the solution idea more concretely, we start with an exemplary use case of synchronising Java code (technically represented by its abstract syntax tree) with its documentation. A small example instance is depicted in Fig. 1. It consists of two classes for geometric figures - Rectangle and 
Parallelogram - and some basic methods and attributes that characterise their side lengths, areas and extents. When the last synchronisation took place, both Parallelogram and Rectangle had attributes sideA and sideB for the length of their respective sides, no further functionality was provided. Two conflicting refactorings take place now to improve the software system. In the Java code, the developer decides to delete the class Parallelogram, as it appears to be an unnecessary generalisation of the Rectangle. Furthermore, two methods getArea and getExtent are defined to compute the respective properties of the shape. In the documentation, in contrast, the developer introduces an inheritance relation between Rectangle and Parallelogram, such that the respective attributes can be deleted from the Rectangle as they are inherited. As the computation of the extent is equal for both classes, this method is added to the Parallelogram. The method getArea, in contrast, is added to the Rectangle, because an additional height attribute would be necessary for the Parallelogram to compute its area. Apparently, these changes are partly in conflict with each other: On the one hand, the Parallelogram cannot be deleted and modified at the same time. On the other hand, assuming that Parallelogram shall be kept, the method getExtent is present in both classes, which might be problematic for programming languages that do not allow method overriding ${ }^{1}$.

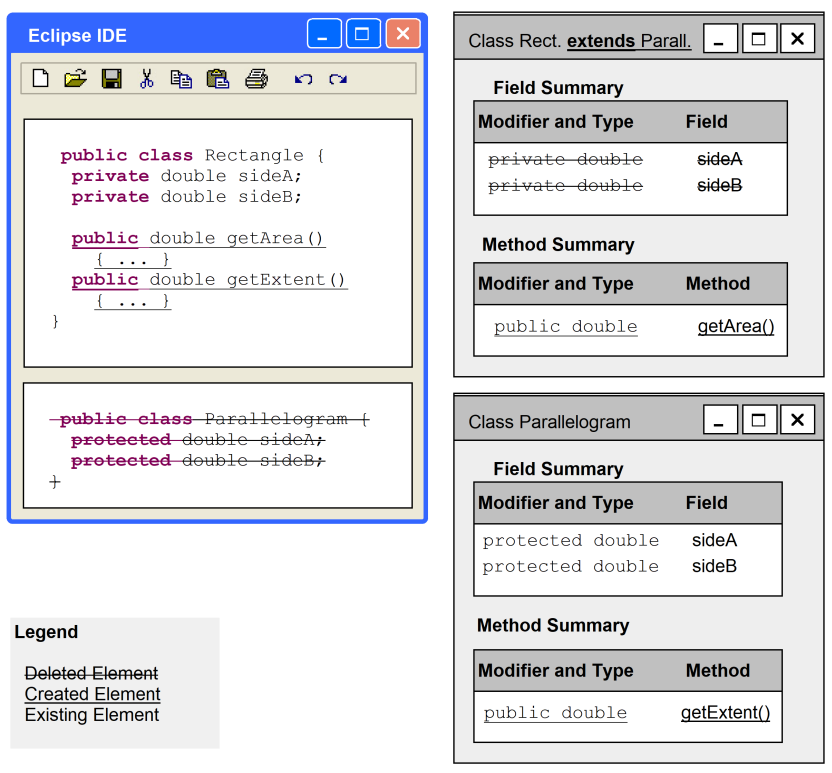

Figure 1: Conflicting changes code and documentation

\subsection{Overview of the Approach}

The goal of our approach is to automatically compute a solution, in which the two models are consistent and which incorporates as many user edits as possible while avoiding further system modifications as far as possible, because this appears to be confusing for the systems' users [7]. While the search-space is constructed in

${ }^{1} \mathrm{~A}$ similar conflict could also be constructed for attributes, which is not allowed in most programming languages accordance with the ILP-based approach [32], we adapt both singleand multi-objective optimisation heuristics to the problem domain to analyse whether there is a reasonable trade-off between solution quality and runtime performance. Despite the fact that the problem is inherently multi-dimensional, an aggregation into a single objective should be considered as an efficient and simple alternative to compute sufficiently good solutions. It is crucial, however, to use appropriate weights for the scalarisation, as large parts of the pareto front are omitted ${ }^{2}$, depending on the problem size and the number of objectives. This aspect was priorly circumvented by leaving the determination of weighting parameters to future work. We therefore propose weightings as part of our evaluation (cf. Sect. 4). As a result of studying related approaches in Sect. 2, we use GA and SA for single- and NSGA-II for multi-objective optimisation.

The different optimisation objectives become apparent when revisiting the example instance shown in Fig. 1. Ideally, all changes applied by one of the involved developers should be preserved, such that the synchronised solution consists (only) of all elements which were recently added or remained unchanged since the last synchronisation took place, whereas none of the deleted elements should be kept in the result. This, however, is hardly achievable when consistency between the two models is demanded as well. Based on these observations, the involved model elements can be partitioned into four sets, while each of them represents one optimisation objective:

- Unchanged elements: Elements that are not affected by any change should be preserved, if possible.

- Delete delta: Elements that are deleted in one model are in the first instance marked to be deleted. These markings should turn into actual deletions after synchronisation. This applies to the class Parallelogram and its attributes in the Java model, for instance.

- Create delta: New elements in one model should be kept with high priority, which makes it necessary to propagate these changes to the other model as well. The inheritance relation introduced in the documentation model, for example, should be reflected in the code as well.

- Induced delta: New model elements require the creation of further elements in the other model, but their number should be as small as possible. It would, for instance, be possible to propagate the creation of the method getArea cross-wise to the respective other model, ending up in duplicating the method. Instead, it is preferable to link the two new model elements to each other.

To formalise the synchronisation problem, we use TGGs [31], in accordance with the priorly presented ILP-based approach [32], from which the problem definition can be taken over to a large extent. TGGs are a rule-based means of (bidirectional) model transformation, i.e., rules for different operations, such as concurrent model synchronisation in this case, can be directly derived from a declarative specification. Our optimisation strategy operates on the level of TGG rules, which means that each potential application of a rule is associated to a variable for the optimisation problem. This makes it possible to construct a search space of manageable size.

${ }^{2}$ cf. Coello Coello et al. [9] for an in-depth discussion of scalarising multi-objective optimisation problems 
It would also be possible to optimise on element level [8], which leads to a substantially larger search space and consequently to performance losses [2], as further constraints have to be introduced that guarantee basic graph properties and correct typing of model elements. Due to the scope of the paper, only the basic ideas of the TGG formalism are introduced, the interested reader is referred to Anjorin et al. [3] for further details.

TGGs express the consistency relation between two models by introducing a third correspondence model that connects semantically interrelated elements of both models. The resulting model triples conform to metamodels, which are depicted in Fig. 2 for the example use case of synchronising Java code with its documentation. On the left-hand side, it is shown that a Java model consists of Classes that can form an inheritance hierarchy via the recursive subtypes relation. Classes can have arbitrarily many Fields (i.e. Attributes) and Methods. All entities are (non-uniquely) identified by a name attribute of type String. On the right-hand side, the documentation metamodel shows that Documents consist of a (possibly empty) list of Entries and can refer to other documents (href relation). The diamonds in between the two models represent the nodes of the correspondence metamodel, expressing that a Java Class, for instance, can correspond to a Document in the documentation model, and the other way round.

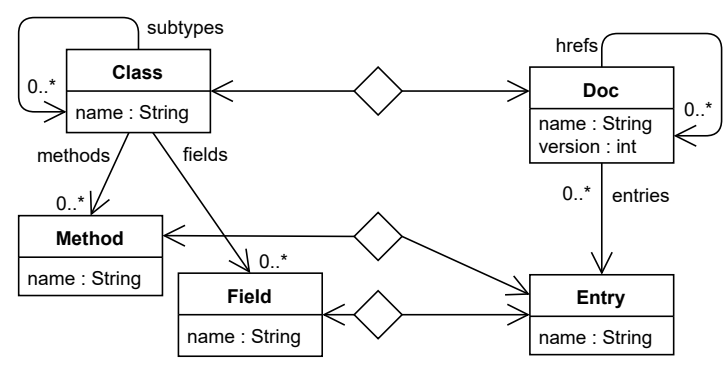

Figure 2: Metamodel: Java code and its documentation

Now, suppose that there are four rules describing the consistency relation between Java code and documentation models as follows:

- ClassToDoc: For each Class $c$, there has to be a corresponding Doc $d$

- SubClassToDoc: For each Class $c 2$ which is a subtype of another Class $c 1$, there must be a Doc $d 2$ with a hyper-reference to the Doc $d 1$ that corresponds to $c 1$

- FieldToEntry: For each Field $f$ of a Class $c$, there has to be an Entry $e$ of a Doc $d$, whereby $c$ and $d$ must correspond to each other

- MethodToEntry: For each Method $m$ of a Class $c$, there must be an Entry $e$ of a Doc $d$, whereby $c$ and $d$ must correspond to each other

These descriptions reflect the declarative definition of rules, which have to be operationalised to be applicable for model synchronisation. Depending on the operation, rule application candidates are collected by means of pattern matching on given input models, out of which the search space is constructed later on. We distinguish between four (basic) types of operations:
- CO (Check Only): Comprises existing elements of all three models in order to mark them as consistent

- CC (Correspondence Creation): Connects new elements in the Java and the documentation model with a correspondence, if this is possible with respect to one of the rules

- FWD (Forward Transformation): Translates a new element of the Java model to the documentation model along with a correspondence node

- BWD (Backward Transformation): Analogous to FWD in the opposite direction

After searching for rule application candidates on the example instance of Fig. 1, a list as shown in Tab. 1 can be constructed. AC denotes the application candidate for the rule named in the second column. The concerned elements of Java and documentation model are listed in the third column (whereby $(\mathrm{R})$ and $(\mathrm{P})$ refer to fields and methods of the classes Rectangle and Parallelogram, respectively), the type of operation in the fourth. Apparently, there are also interdependencies between the rule application candidates: To apply, e.g., the rules FieldToEntry for the side $a$ of the Rectangle $\left(r_{4}\right)$, it is necessary that the Rectangle itself is also part of the solution $\left(r_{1}\right)$. Furthermore, it is not possible to choose all candidates to construct the synchronisation solution because some elements are part of multiple rule applications. The Parallelogram, e.g., can be either treated as a standalone class $\left(r_{2}\right)$ or as a subclass of Rectangle $\left(r_{3}\right)$. Similarly, the method getArea of the Rectangle, which was created in both models, can either be translated twice in both directions $\left(r_{6}\right.$, $r_{7}$ ), or the nodes of both models can be connected with a correspondence $\left(r_{8}\right)$. These interdependencies are encoded as constraints that restrict the feasible region of the search space.

An example for a feasible solution which satisfies all constraints would be to choose $r_{1}, r_{2}, r_{4}, r_{5}, r_{8}, r_{9}, r_{10}, r_{11}$ and $r_{12}$. In this case, Rectangle and Parallelogram would be kept as independent classes which both have their attributes sideA and sideB. Furthermore, the getArea methods of Rectangle are linked to each other (by applying $r_{8}$ instead of $r_{6}$ and $r_{7}$ ), while the new getExtent methods are translated to the respective other models. The optimality of a solution clearly depends on the weighting of objectives: Giving a higher priority to preserving deletions, the Parallelogram class and all attributes would be omitted, implying that adding an inheritance relation and a new method getExtent is not possible in this solution. For a detailed description of the constraint generation and objective function construction, the reader is referred to [32].

\begin{tabular}{|c|c|c|c|c|c|}
\hline AC & Rule & Elements & Op. & Implies & Excludes \\
\hline$r_{1}$ & ClassToDoc & Rectangle & CO & - & - \\
$r_{2}$ & ClassToDoc & Parallelogram & CO & - & $r_{3}$ \\
$r_{3}$ & SubClassToDoc & Parallelogram & BWD & $r_{1}$ & $r_{2}$ \\
$r_{4}$ & FieldToEntry & sideA (R) & CO & $r_{1}$ & $r_{3} \wedge r_{10}$ \\
$r_{5}$ & FieldToEntry & sideB (R) & CO & $r_{1}$ & $r_{3} \wedge r_{11}$ \\
$r_{6}$ & MethodToEntry & getArea (R) & FWD & $r_{1}$ & $r_{8}$ \\
$r_{7}$ & MethodToEntry & getArea (R) & BWD & $r_{1}$ & $r_{8}$ \\
$r_{8}$ & MethodToEntry & getArea (R) & CC & $r_{1}$ & $r_{6} \vee r_{7}$ \\
$r_{9}$ & MethodToEntry & getExtent (R) & FWD & $r_{1}$ & $r_{3} \wedge r_{12}$ \\
$r_{10}$ & FieldToEntry & sideA (P) & CO & $r_{2} \vee r_{3}$ & $r_{3} \wedge r_{4}$ \\
$r_{11}$ & FieldToEntry & sideB (P) & CO & $r_{2} \vee r_{3}$ & $r_{3} \wedge r_{5}$ \\
$r_{12}$ & MethodToEntry & getExtent (P) & BWD & $r_{2} \vee r_{3}$ & $r_{3} \wedge r_{9}$ \\
\hline
\end{tabular}

Table 1: Search space for the example instance 


\subsection{Adaptation of Meta-Heuristics}

In the following, we will present how solution candidates can be encoded, evaluated and varied throughout the search process for the three chosen meta-heuristics. The encoding of solution candidates and operators can be kept mostly similar for all algorithms, differences are pointed out explicitly.

3.3.1 Encoding of Solution Candidates. Solution candidates must provide information about which rule applications contribute to the synchronisation solution. Therefore, it seems suitable to encode them as a binary string, in which each bit is associated to one rule application candidate. The bit is set to 1 iff the rule application is selected for the synchronisation solution. The initialisation of the population for GA and NSGA-II is straightforward as random binary strings are generated. This would also be possible to initialise SA, which only operates on a single solution in each step. However, as it is beneficial to start with a fairly good solution, the SA is initialised with a string of zeros, which represents the empty triple graph because no rule application is selected. Although the solution is usually far below the optimum, it is guaranteed not to violate any constraint by definition and is therefore part of the feasible region. The suggested solution for the example instance (cf. Sect. 3.2), would be encoded as 110110011111 .

3.3.2 Treatment of the Infeasible Region. The chosen form of encoding solution candidates adds a noticeably large set of invalid solutions to the search space, i.e., solutions that violate at least one of the correctness constraints. The lack of a rule application which is implied by others immediately leads to invalid sequences, which violates at least one constraint (Tab. 1, column 5) and is therefore located in the infeasible region. In turn, a superfluous rule application might translate elements twice, which is also prohibited by constraints (Tab. 1, column 6). In the running example, each solution candidate starting with 111 would violate the constraint that mutually excludes $r_{2}$ and $r_{3}$, for instance. Instead of rejecting invalid solutions immediately, a penalty is introduced to the objective function that guarantees that such solutions cannot have a higher fitness than the empty triple graph, which is always valid (cf. Sect. 3.3.1). Similar to using mutation or perturbation (cf. Sect. 3.3.4), this technique is beneficial for escaping local optima [25].

3.3.3 Fitness Function. The quality of solution candidates is assessed using the fitness function, which also serves as a basis for selecting individuals for recombination. While NSGA-II allows arbitrarily many objective functions, these have to be scalarised to a single function for GA and SA. For NSGA-II, we define four objectives that resemble the optimisation goals listed in Sect. 3.2. Let $M$ denote the model triple to be synchronised, and let $R$ be the set of rule applications that contribute to the synchronisation solution. unchanged, dltDelta, crtDelta and indDelta denote the respective partitions of the model elements as described in Sect. 3.2. The element sets $\operatorname{tr}(r)$ and $\operatorname{cr}(r)$ contain nodes and edges which are translated and created by a rule application $r$, resulting in the following objective functions:

$$
\begin{aligned}
& \max . \sum_{r \in R} \mid \operatorname{tr}(r) \cap \text { unchanged }(M)\left|\quad \min . \sum_{r \in R}\right| \operatorname{tr}(r) \cap \operatorname{dltDelta}(M) \mid \\
& \max . \sum_{r \in R}|\operatorname{tr}(r) \cap \operatorname{crtDelta}(M)| \quad \min . \sum_{r \in R}|\operatorname{cr}(r) \cap \operatorname{indDelta}(M)|
\end{aligned}
$$

For GA and SA, these four functions must be aggregated into a single function with suitable weightings, choosing negative weighting parameters for the minimisation objectives, such that the resulting weighted sum can be maximised. Following the notation of the ILP-based approach, we weight the delete delta with $\alpha<0$, the create delta with $\beta>0$ and the induced delta with $\gamma<0$. The function for the unchanged part of the model serves as norm with weight 1 . Suitable values for $\alpha, \beta$ and $\gamma$ are investigated on in the second part of the evaluation (Sect. 4).

3.3.4 Mutation and Perturbation. The mutation operator is a crucial factor for genetic algorithms to add diversity to the population. A similar concept for SA is perturbation, where neighbouring solutions are generated in each iteration by modifying the previous solution. As solution candidates are encoded as binary strings, it is advisable to use bit flips at random positions of the genotype. For the phenotype, mutation and perturbation add or remove rule applications from the solution candidate. The probability for each bit to be flipped is set to $1 / N$, whereby $N$ denotes the number of bits, such that the expected number of bit flips is 1 per individual. Regarding the running example, a possible outcome would be to flip the ninth bit from 1 to 0 . As a result, $r_{9}$ is removed from the solution, such that the method getExtent is not created in the class Rectangle.

3.3.5 Crossover. With the crossover operator, promising genetic features of parent individuals shall be recombined to generate offspring with an even higher fitness value. For GA and NSGA-II, we use single-point crossover, which splits the bit strings $A$ and $B$ representing the parent individuals at a random position into two parts $A^{\prime} / A^{\prime \prime}$ and $B^{\prime} / B^{\prime \prime}$. The offspring is then generated by concatenating $A^{\prime} / B^{\prime \prime}$ and $B^{\prime} / A^{\prime \prime}$, respectively. SA, in contrast, does not have such an operator as only one current solution exists at each point of time. Suppose that the example solution (cf. Sect. 3.3.1) is recombined with another solution that introduces an inheritance relation between Rectangle and Parallelogram, and in turn removes both attributes and the method getExtent from the Rectangle (encoded as 101000010111). When splitting after the sixth bit, the getExtent method $\left(r_{9}\right)$ is shifted from the first to the second individual. In this case, the crossover does not generate fitter offspring, which is likely in case the parent individuals have a high fitness value already.

3.3.6 Selection and Cooling. For the genetic algorithms GA and NSGA-II, the computed fitness of individuals is used to decide which of them are selected to enter the next generation. We use binary tournament selection, i.e., two individuals are randomly and repeatedly picked from the population and the one with the better fitness is selected. In SA, in contrast, a "temperature", which is continuously decreased, defines the probability for accepting worse solutions during the iterative search. This leads to a compromise between exploring the search space in the beginning and exploiting it towards the end. For a concrete software implementation, several variants of this meta-heuristic are possible. We followed the proposition of Bill et al. [6], in which neighbours are generated out of a working solution that is reset to the best solution found when no improvement could be achieved for a pre-defined number of iterations. 


\section{EVALUATION}

To assess the applicability of our approach for realistic use cases, we implemented a heuristic concurrent synchronisation algorithm based on the three meta-heuristics discussed in this paper (GA, NSGA-II and SA). In particular, the following three research questions shall be answered by our evaluation:

RQ1 Is it feasible in practice to present the set of pareto-optimal solutions to users, such that they can choose one of them to synchronise concurrent updates?

RQ2 How can suitable parameters $\alpha, \beta$ and $\gamma$ be determined to scalarise multiple objectives for a concrete use case?

RQ3 To which extent are heuristic solutions applicable in practice regarding runtime behaviour and solution quality?

Experimental setup and basic parametrisation: Our approach was implemented as an extension of the model transformation tool eMoflon ${ }^{3}$, which is an open source Java-based Eclipse plug-in with several interfaces for external tools and frameworks. GA and NSGA-II were integrated into the tool via the MOEA Framework ${ }^{4}$, whereas SA was implemented manually. We followed the SA implementation proposed by Bill et al. [6] parametrised with $c_{c}=3$ and $c_{b}=10$. For a comparison to the baseline approach, the Gurobi optimiser $^{5}$ (Version 8.1.1) was used for ILP solving. eMoflon uses $\mathrm{Neo}_{4} \mathrm{j}^{6}$ (Version 4.2.1) as a graph database for storing (meta-)models. All performance tests were executed on a standard notebook with an Intel Core i7 (1.80 GHz), 16GB RAM, and Windows 10 64-bit as operating system. An installation of Eclipse IDE for Java and DSL Developers, version 2019-09 with Java Development Kit (JDK) version 13 was used. 4GB memory each were allocated to the JVM running the tests and to the graph database Neo4j. For each configuration, only the time required for the optimisation step was measured, because other tasks such as graph pattern matching are independent of the optimisation algorithm. Each test run was repeated 30 times to minimise the bias introduced by outliers, the median was taken as final value. Due to space limitations, only a summary of the results can be provided, a detailed overview of the examples and collected data is available online ${ }^{7}$.

RQ1: Analysis of the pareto front: We set up small use case in the setting of the running example, i.e., a concurrent synchronisation problem for Java code and its documentation. Independent of the concrete syntax of source and target models, an overview of the use case is provided in Fig. 3 in form of a UML class diagram. For four geometric figures, it shall be possible to compute their area and extent out of their attributes. The initial model shall therefore be improved using five refactorings, which are partly in conflict with each other. Elements which are deleted or created via a refactoring are depicted in red (--) and green $(++)$, respectively, all other elements remain unchanged. Furthermore, the elements are annotated with the number of the refactoring which modifies them. Possible refactorings are the introduction of an inheritance relation between Square and Parallelogram (1), Square and Diamond (2),

\footnotetext{
${ }^{3}$ https://emoflon.org/

${ }^{4}$ http://moeaframework.org/

${ }^{5}$ https://www.gurobi.com/

${ }^{6} \mathrm{https} / / /$ neo4j.com/

${ }^{7}$ https://drive.google.com/file/d/1elGTTplm6RKm8IQaiNzZyP57V8Rrj_90/view
}

the enrichment of Diamond with further attributes (3), a new inheritance relation between Rectangle and Parallelogram (4) and the deletion of the class Parallelogram (5).

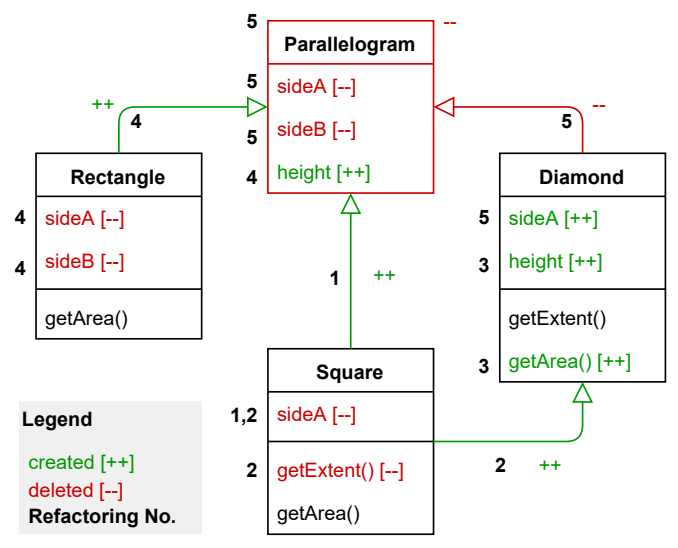

Figure 3: Metamodel: Java code and its documentation

Each subset of three refactorings was applied on the initial instance (two on the Java model, one on the documentation model), such that at least one conflict was induced. The refactorings were distributed such that those two on the Java model are not conflicting. For the resulting ten variants, the set of pareto-optimal solutions was computed using NSGA-II with one million iterations each. Regarding the number of retrieved solutions, the median for all test runs and variants (i.e. refactoring combinations) was 13, reaching from 10 to 33 for single variants. The mean was 16.323, reaching from 8.900 to 33.333 , and the standard deviation 9.806 , reaching from 1.661 to 8.768 . The median runtime for all test runs and variants was $32.047 \mathrm{~s}$, the mean was $32.375 \mathrm{~s}$ and the standard deviation $2.733 \mathrm{~s}$.

The experiment reveals some limitations for working with a multi-objective approach in practice. Without further support, it is not feasible for a user to choose from the complete set of paretooptimal solutions. Although the setting of the use case is not generalisable to arbitrary synchronisation problems, it is very likely that for realistic model sizes, the set of pareto-optimal solutions is substantially larger. Furthermore, the number of evaluations was chosen with the goal of being sufficiently high for retrieving the whole set of pareto-optimal solutions in each test run, leading to an average runtime of about half a minute for solving a single synchronisation problem, which is not acceptable for practical applications. However, there was a high variance in the size and composition of the retrieved solution sets, indicating that substantially more resources would be necessary to find the whole set in a single run. As a result, we formed a union of all pareto-optimal solution sets for each variant and continued with these sets to answer RQ2. After removing duplicates and solutions which are dominated in the union of all sets for a variant, 451 solutions were determined, i.e. 45.1 per variant (median: 52 ).

RQ2: Parameter determination: As the experiment to answer RQ1 shows that it is not feasible to let the user choose from all pareto-optimal solutions even for small model sizes, a single objective function must be constructed to make the approach both 
efficient and applicable in practice. However, it is hardly possible to determine generally valid parameter values for aggregating the four involved objectives into a single function, as their choice depends on the underlying TGG. If, for example, the involved models are extensively nested, a higher absolute value for $\alpha$ is required as elements on top of such a hierarchical structure can never be deleted otherwise, in contrast to rather flat model structures for which an $\alpha$ value closer to 0 might be sufficient. Therefore, we restrict ourselves to an exemplary determination of parameter values for the example TGG used throughout this paper, and simultaneously propose a method to find suitable values for other use cases as well. It is furthermore not possible to let users estimate suitable values for those parameters directly, because their meaning is very abstract and even model transformation experts lack an intuition for the consequences of particular parameter choices. Therefore, we follow an indirect approach of empirically assessing the paretooptimal solutions for the use case of RQ1. 9 undergraduate students with sound programming skills and basic knowledge on MDE were asked to rate the quality of different pareto-optimal solutions on a scale reaching from 0 (no agreement) to 10 (total agreement). The rating shall be based on to which extent the synchronisation solution reflects the intention behind the applied refactorings. As the entire solution set is even too large for this study, scalarisations for different value combinations were computed, and the solution set was restricted to those candidates which are optimal for at least one scalarisation. The choice was made in accordance with the value range defined in prior work $(\alpha<0, \beta>0, \gamma<0)$, and the following preliminary considerations were taken into account:

- $\alpha$ should be at most -1 , such that for each deleted element in one model, it is possible to delete an element in the other model without decreasing the objective function value.

- $\beta$ should be at least 1 , as it is at least as important to preserve added elements as to preserve unchanged elements.

- $\gamma$ should be $\in[-1 ; 0)$, because it should serve as a tie breaker in cases where it is possible to allocate created elements to each other instead of translating them anew (cf. Sect. 3.2). For each new element in one model, it should be possible to add a respective element in the other model without decreasing the objective function value.

We observed that further decreasing $\alpha$ beyond -4 or increasing $\beta$ or $\gamma$ beyond 4 and -0.6 , respectively, in isolation does not influence which of the pareto-optimal solutions is optimal for the scalarisation. Therefore, the chosen value ranges were $\alpha \in\{-1,-2,-3,-4\}$, $\beta \in\{1,2,3,4\}$ and $\gamma \in\{-0.6,-0.8,-1\}$, resulting in 48 different scalarisations. The mean ${ }^{8}$ participant rating was allocated to the parameter combination, for which the respective solution is optimal. The average rating of all 10 variants was taken as rating for the respective parameter combination.

The results are visually depicted in Fig. 4, where a cuboid represents the investigated parameter value range and its colouring the average rating of the respective parameter combination. The observed upper and lower bounds correspond to pure blue (9) and pure red (4), while values in between are represented by a proportional mix of these two colours. The front faces in the left/right

${ }^{8}$ Using the median instead of the mean appears to be inappropriate here as the loss of precision is more harmful than the effect of statistical outliers on a scale from 0 to 10 . diagram correspond to ratings for $\gamma=0.6 / 1.0$. One can observe that choosing $\gamma$ closer to 0 leads to better ratings, only a combination with $\alpha$ values close to 1 seem to be problematic. This problem gets more severe when choosing $\gamma=1$, here only combinations with $\alpha<-2$ and $\beta>2$ lead to good ratings. Interestingly, the choice of $\beta$ seems to be more important for combinations with $\gamma$ close to one, which indicates that the ratio between $\beta$ and $\gamma$ has to be "large enough". This seems reasonable due to the interplay of propagating user additions and avoiding unnecessary model extensions. Similarly, propagating deletions (weighted with $\alpha$ ) and preserving unchanged elements (weighted with 1 ) are especially conflicting, which means that the effect of changing $\alpha$ should be quite independent of the choice of $\beta$ and $\gamma$. This claim is also supported by our measurements. Finally, considering the right diagram in which $\gamma=1$ holds for the front face, it seems to be useful to keep the ratio between $\beta$ and $\gamma$ approximately equal to $\alpha$ as the diagonal from $(-1 / 1 /-1)$ to $(-4 / 4 /-1)$ cuts the front face almost into symmetric halves.
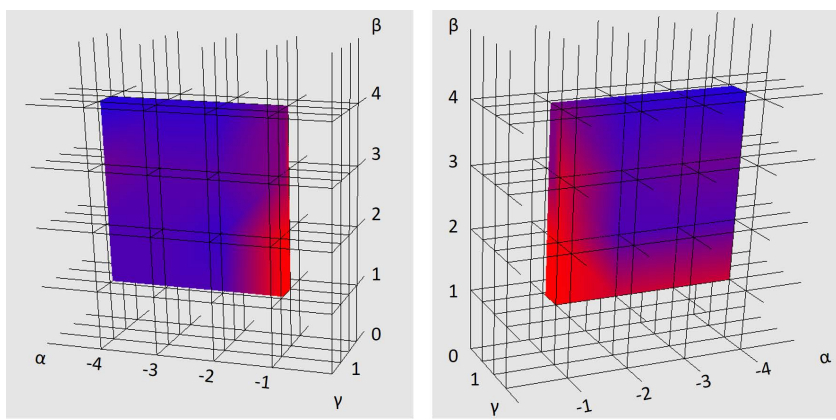

Figure 4: Average ratings for parameter combinations

RQ3: Runtime and quality analysis: To analyse how the runtime performance of GA and SA compare to ILP, we ran the respective algorithms on synthetic model instances with linearly increasing sizes from 460 to 2141 elements. For simulating edit operations, create and delete markers were randomly assigned to $20 \%$ of the model elements each, resulting in a comparably high change density. We used the TGG presented throughout this paper in its extended version as specified in [15, 32]. Due to space limitations, the results are presented in an aggregated form, details are available online ${ }^{9}$. For GA, a population size of 100 was used. SA was run with linear cooling from the start temperature $T_{0}$ down to 0 and a geometric cooling using a factor of 0.98 , with a re-heating to $T_{0}$ as soon as $T$ falls below a threshold of 1 . The measurements did not show any statistically significant difference for the cooling schemes regarding runtime or solution quality, therefore we restrict ourselves to the presentation of the geometric variant. As a stop criterion, we defined a limit for the number of iterations per test run, which is equal for all heuristics in order to guarantee comparability. The limit depends linearly on the length of the bit string, such that a larger number of iterations is granted for larger models. For SA, also test runs with only $10 \%$ of the iterations were conducted ("short runs") to analyse the effect of this criterion.

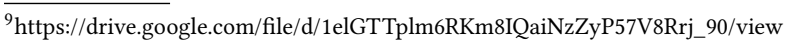


The median runtime values per model instance and algorithm are depicted in Fig. 5. As expected, the runtime grows slightly super-linear with the model size, because the stop criterion depends linearly on the model size and the generation of new solution candidates becomes more time-consuming for longer bit strings. For SA and GA, the observed runtime behaviour did not show any significant differences. Interestingly, the ILP baseline outperformed these two heuristics, showing better scalability characteristics for larger models, similar to the short run version of SA.

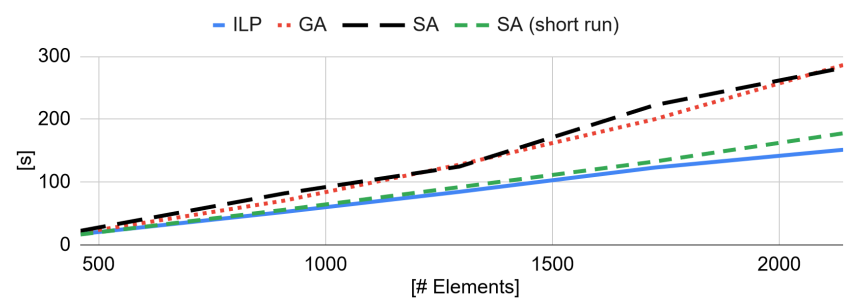

Figure 5: Runtime analysis for different model sizes

To measure the solution quality, the objective function value of the returned solution was used. In comparison to the solution determined by ILP solving, which is guaranteed to be optimal, it is possible to assess how close to the optimum heuristic solutions are on average. The median objective function values are shown in Fig. 6. For SA, the 95\% confidence interval is further displayed with dotted lines. GA found feasible solutions only in 16 test runs for the smallest model size and in no test run for the largest two model sizes, such that the heuristic is left out in this diagram. SA, in contrast, could determine solutions with objective function values of $81.6 \%-94.1 \%$ of the optimum, taking the minimum and maximum of the $95 \%$ confidence intervals of all model sizes. The short run version of SA was clearly outperformed, though. These results especially the weak performance of GA - are unexpected in the first place, which is why we assume that the search space is infeasible for the very most part. To support this assumption, we randomly sampled solutions using the same number of iterations as for GA and SA. In none of the test runs (consisting of approx. 1.5 million iterations for the smallest and 7.5 million for the largest model), a feasible solution was found. With respect to this observation, it seems to be unrealistic to expect a heuristic algorithm to reliably return a near-optimal solution.

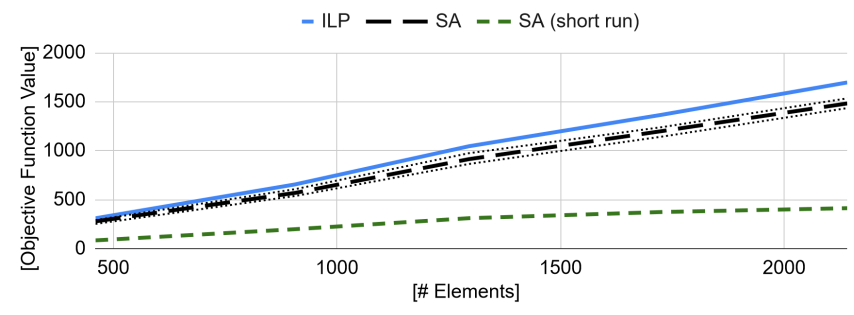

Figure 6: Quality analysis for different models sizes

Summary: Revisiting our research questions, one can state that even for small examples, the pareto front contains too many solutions to be entirely presented to the user, such that a scalarisation to a single objective function is necessary [RQ1]. With help of an empirical user study, a method to determine suitable parameter values for $\alpha, \beta$ and $\gamma$ was presented for the use case of RQ1, which is applicable to other use cases, too [RQ2]. A performance evaluation has shown that GA is not an adequate heuristic in this setting as it often fails to find even a feasible solution. SA performed much better (conforming to the observation of Bill et al. [6] for search spaces with a large infeasible region), whereas near-optimal solutions could not be determined faster than the optimal solution via ILP solving. In general, the measured solution quality of $81.6 \%$ $94.1 \%$ for SA implies that a noticeable part of the changes is unnecessarily dropped, which will not be perceived as an acceptable solution for most users. In summary, we recommend to stick to the ILP-based approach, while the parameter analysis for answering RQ2 helps to configure the respective optimisation function.

Threats to validity: Although the approach was tested in depth for one example, results for RQ2 and RQ3 may differ when applying it on other synchronisation problems. The number of participants for determining suitable parameters was low, such that the results might be biased by personal preferences. Furthermore, the chosen use case was synthetic and only involved small models, such that the design of the user study might become infeasible for other synchronisation problems and larger models. Although there was a large performance gap between ILP, SA and GA in our experiments, it is possible that another operator implementation or parametrisation of the meta-heuristics could lead to more promising results that outperform the exact solution. As we rely on a standard framework for GA and NSGA-II and the Gurobi solver for ILP, the efficiency of the implementation in external components has an influence on the runtime performance as well.

\section{CONCLUSION AND FUTURE WORK}

We presented a heuristic approach to concurrent model synchronisation, which is an important but insufficiently studied problem in the area of MDE. Based on a predetermined set of TGG rule applications, a search space is constructed in which each solution candidate represents a possible synchronisation strategy via a subset of rule applications. By adapting (genetic) operators to the problem domain, an implementation involving three single- and multi-objective optimisation heuristics is presented based on a model transformation tool under active development. However, the use of heuristics did not yield the desired performance improvements due to the large portion of infeasible solutions. Therefore, it is still recommendable to use exact solvers in this setting to obtain solutions of satisfactory quality. A generalisable methodology was proposed to configure the objective function for a concrete applications scenario.

To carry this line of research forward, we plan to analyse further use cases involving other TGGs and larger model instances to see in which scenarios recommendable parameters differ from the results of this study. Furthermore, an implementation of further metaheuristics, which have proven to be beneficial for solution spaces with a large infeasible region, might be promising to improve the overall runtime performance. Finally, we are working on improving the user involvement in resolving synchronisation conflicts, such that the search for an optimal solution is at least partially guided by a human user who is able to make case-specific decisions. 


\section{ACKNOWLEDGMENTS}

This work was partially supported by the North Rhine Westphalian Ministry of Economic Affairs, Innovation, Digitalisation and Energy (MWIDE) through the Pro-LowCode project (005-2011-0022). Also, we would like to thank Anthony Anjorin (IAV GmbH) and Lars Fritsche (TU Darmstadt) for their helpful feedback.

\section{REFERENCES}

[1] Hani Abdeen, Dániel Varró, Houari A. Sahraoui, András Szabolcs Nagy, Csaba Debreceni, Ábel Hegedüs, and Ákos Horváth. 2014. Multi-objective optimization in rule-based design space exploration. In International Conference on Automated Software Engineering, ASE '14, Vasteras, Sweden - September 15 - 19, 2014, Ivica Crnkovic, Marsha Chechik, and Paul Grünbacher (Eds.). 289-300. https://doi.org/ 10.1145/2642937.2643005

[2] Anthony Anjorin, Thomas Buchmann, Bernhard Westfechtel, Zinovy Diskin Hsiang-Shang Ko, Romina Eramo, Georg Hinkel, Leila Samimi-Dehkordi, and Albert Zündorf. 2020. Benchmarking bidirectional transformations: theory, implementation, application, and assessment. Softw. Syst. Model. 19, 3 (2020), 647-691. https://doi.org/10.1007/s10270-019-00752-x

[3] Anthony Anjorin, Erhan Leblebici, and Andy Schürr. 2015. 20 Years of Triple Graph Grammars: A Roadmap for Future Research. Electron. Commun. Eur. Assoc. Softw. Sci. Technol. 73 (2015). https://doi.org/10.14279/tuj.eceasst.73.1031

[4] Wesley K. G. Assunção, Silvia R. Vergilio, and Roberto E. Lopez-Herrejon. 2017. Discovering Software Architectures with Search-Based Merge of UML Model Variants. In Mastering Scale and Complexity in Software Reuse - 16th International Conference on Software Reuse, ICSR 2017, Salvador, Brazil, May 29-31, 2017, Proceedings, Goetz Botterweck and Cláudia Werner (Eds.). 95-111. https://doi.org/10.1007/978-3-319-56856-0_7

[5] Ameni ben Fadhel, Marouane Kessentini, Philip Langer, and Manuel Wimmer 2012. Search-based detection of high-level model changes. In 28th IEEE International Conference on Software Maintenance, ICSM 2012, Trento, Italy, September 23-28, 2012. 212-221. https://doi.org/10.1109/ICSM.2012.6405274

[6] Robert Bill, Martin Fleck, Javier Troya, Tanja Mayerhofer, and Manuel Wimmer. 2019. A local and global tour on MOMoT. Softw. Syst. Model. 18, 2 (2019), 10171046. https://doi.org/10.1007/s10270-017-0644-3

[7] James Cheney, Jeremy Gibbons, James McKinna, and Perdita Stevens. 2017. On principles of Least Change and Least Surprise for bidirectional transformations. 7. Object Technol. 16, 1 (2017), 3:1-31. https://doi.org/10.5381/jot.2017.16.1.a3

[8] Antonio Cicchetti, Davide Di Ruscio, Romina Eramo, and Alfonso Pierantonio. 2010. JTL: A Bidirectional and Change Propagating Transformation Language. In Software Language Engineering - Third International Conference, SLE 2010, Eindhoven, The Netherlands, October 12-13, 2010, Revised Selected Papers, Brian A. Malloy, Steffen Staab, and Mark van den Brand (Eds.). 183-202. https://doi.org/ 10.1007/978-3-642-19440-5 11

[9] Carlos Artemio Coello Coello, Gary B. Lamont, and David A. van Veldhuizen. 2007. Evolutionary algorithms for solving multi-objective problems, Second Edition.

[10] Hoa Khanh Dam, Alexander Egyed, Michael Winikoff, Alexander Reder, and Roberto E. Lopez-Herrejon. 2016. Consistent merging of model versions. F. Syst. Softw. 112 (2016), 137-155. https://doi.org/10.1016/j.jss.2015.06.044

[11] Hoa Khanh Dam, Alexander Reder, and Alexander Egyed. 2014. Inconsistency Resolution in Merging Versions of Architectural Models. In 2014 IEEE/IFIP Conference on Software Architecture, WICSA 2014, Sydney, Australia, April 7-11, 2014. 153-162. https://doi.org/10.1109/WICSA.2014.31

[12] István Dávid, Joachim Denil, Klaas Gadeyne, and Hans Vangheluwe. 2016. Engineering Process Transformation to Manage (In)consistency. In Proceedings of the 1st International Workshop on Collaborative Modelling in MDE (COMMitMDE 2016), St. Malo, France, October 4, 2016, Henry Muccini, Ivano Malavolta, Sebastien Gerard, and Dimitris S. Kolovos (Eds.). 7-16. http://ceur-ws.org/Vol-1717/paper5.pdf

[13] Kalyanmoy Deb, Samir Agrawal, Amrit Pratap, and T. Meyarivan. 2002. A fast and elitist multiobjective genetic algorithm: NSGA-II. IEEE Trans. Evol. Comput 6, 2 (2002), 182-197. https://doi.org/10.1109/4235.996017

[14] Csaba Debreceni, István Ráth, Dániel Varró, Xabier De Carlos, Xabier Mendialdua, and Salvador Trujillo. 2016. Automated Model Merge by Design Space Exploration. In Fundamental Approaches to Software Engineering - 19th International Conference, FASE 2016, Eindhoven, The Netherlands, April 2-8, 2016, Proceedings, Perdita Stevens and Andrzej Wasowski (Eds.). 104-121. https: //doi.org/10.1007/978-3-662-49665-7_7

[15] Lars Fritsche, Jens Kosiol, Adrian Möller, Andy Schürr, and Gabriele Taentzer 2020. A precedence-driven approach for concurrent model synchronization scenarios using triple graph grammars. In Proceedings of the 13th International Conference on Software Language Engineering, SLE 2020, Virtual Event, USA, November 16-17, 2020, Ralf Lämmel, Laurence Tratt, and Juan de Lara (Eds.). 39-55. https://doi.org/10.1145/3426425.3426931

[16] Sebastian J. I. Herzig, Benjamin Kruse, Federico Ciccozzi, Joachim Denil, Rick Salay, and Dániel Varró. 2014. Towards an Approach for Orchestrating Design
Space Exploration Problems to Fix Multi-Paradigm Inconsistencies. In Proceedings of the 8th Workshop on Multi-Paradigm Modeling, MPM@MODELS 2014, Valencia, Spain, September 30, 2014, Daniel Balasubramanian, Christophe Jacquet, Pieter Van Gorp, Sahar Kokaly, and Tamás Mészáros (Eds.). CEUR-WS.org, 61-66. http://ceurws.org/Vol-1237/paper6.pdf

[17] John H. Holland. 1992. Adaptation in Natural and Artificial Systems: An Introductory Analysis with Applications to Biology, Control, and Artificial Intelligence. https://doi.org/10.7551/mitpress/1090.001.0001

[18] Gerti Kappel, Philip Langer, Werner Retschitzegger, Wieland Schwinger, and Manuel Wimmer. 2012. Model Transformation By-Example: A Survey of the First Wave. In Conceptual Modelling and Its Theoretical Foundations - Essays Dedicated to Bernhard Thalheim on the Occasion of His 60th Birthday, Antje Düsterhöft, Meike Klettke, and Klaus-Dieter Schewe (Eds.). 197-215. https://doi.org/10.1007/978-3642-28279-9_15

[19] Marouane Kessentini, Arbi Bouchoucha, Houari A. Sahraoui, and Mounir Boukadoum. 2010. Example-Based Sequence Diagrams to Colored Petri Nets Transformation Using Heuristic Search. In Modelling Foundations and Applications - 6th European Conference, ECMFA 2010, Paris, France, fune 15-18, 2010. Proceedings, Thomas Kühne, Bran Selic, Marie-Pierre Gervais, and François Terrier (Eds.). 156-172. https://doi.org/10.1007/978-3-642-13595-8_14

[20] Marouane Kessentini, Houari A. Sahraoui, and Mounir Boukadoum. 2008. Model Transformation as an Optimization Problem. In Model Driven Engineering Languages and Systems, 11th International Conference, MoDELS 2008, Toulouse, France, September 28 - October 3, 2008. Proceedings, Krzysztof Czarnecki, Ileana Ober, Jean-Michel Bruel, Axel Uhl, and Markus Völter (Eds.). 159-173. https://doi.org/ 10.1007/978-3-540-87875-9 12

[21] Marouane Kessentini, Houari A. Sahraoui, Mounir Boukadoum, and Omar Benomar. 2012. Search-based model transformation by example. Softw. Syst. Model. 11, 2 (2012), 209-226. https://doi.org/10.1007/s10270-010-0175-7

[22] Marouane Kessentini, Wafa Werda, Philip Langer, and Manuel Wimmer. 2013. Search-based model merging. In Genetic and Evolutionary Computation Conference, GECCO '13, Amsterdam, The Netherlands, fuly 6-10, 2013, Christian Blum and Enrique Alba (Eds.). 1453-1460. https://doi.org/10.1145/2463372.2463553

[23] Scott Kirkpatrick, D. Gelatt Jr., and Mario P. Vecchi. 1983. Optimization by Simmulated Annealing. Sci. 220, 4598 (1983), 671-680.

[24] Mathias Kleiner, Marcos Didonet Del Fabro, and Davi De Queiroz Santos. 2013. Transformation as Search. In Modelling Foundations and Applications - 9th European Conference, ECMFA 2013, Montpellier, France, July 1-5, 2013. Proceedings, Pieter Van Gorp, Tom Ritter, and Louis M. Rose (Eds.). 54-69. https: //doi.org/10.1007/978-3-642-39013-5 5

[25] Rudolf Kruse, Christian Borgelt, Christian Braune, Sanaz Mostaghim, and Matthias Steinbrecher. 2016. Computational Intelligence - A Methodological Introduction, Second Edition. Springer. https://doi.org/10.1007/978-1-4471-7296-3

[26] Nuno Macedo and Alcino Cunha. 2013. Implementing OVT-R Bidirectional Model Transformations Using Alloy. In Fundamental Approaches to Software Engineering - 16th International Conference, FASE 2013, Rome, Italy, March 16-24, 2013. Proceedings, Vittorio Cortellessa and Dániel Varró (Eds.). 297-311. https: //doi.org/10.1007/978-3-642-37057-1_22

[27] Usman Mansoor, Marouane Kessentini, Philip Langer, Manuel Wimmer, Slim Bechikh, and Kalyanmoy Deb. 2015. MOMM: Multi-objective model merging. F. Syst. Softw. 103 (2015), 423-439. https://doi.org/10.1016/j.jss.2014.11.043

[28] Fernando Orejas, Elvira Pino, and Marisa Navarro. 2020. Incremental Concurrent Model Synchronization using Triple Graph Grammars. In Fundamental Approaches to Software Engineering - 23rd International Conference, FASE 2020, Dublin, Ireland, April 25-30, 2020, Proceedings, Heike Wehrheim and Jordi Cabot (Eds.). 273-293. https://doi.org/10.1007/978-3-030-45234-6_14

[29] Julia Rubin and Marsha Chechik. 2013. N-way model merging. In foint Meeting of the European Software Engineering Conference and the Symposium on the Foundations of Software Engineering, ESEC/FSE'13, Saint Petersburg, Russian Federation, August 18-26, 2013, Bertrand Meyer, Luciano Baresi, and Mira Mezini (Eds.). 301-311. https://doi.org/10.1145/2491411.2491446

[30] Hajer Saada, Marianne Huchard, Clémentine Nebut, and Houari A. Sahraoui. 2013. Recovering model transformation traces using multi-objective optimization. In 2013 28th International Conference on Automated Software Engineering, ASE 2013, Silicon Valley, CA, USA, November 11-15, 2013, Ewen Denney, Tevfik Bultan, and Andreas Zeller (Eds.). 688-693. https://doi.org/10.1109/ASE.2013.6693134

[31] Andy Schürr. 1994. Specification of Graph Translators with Triple Graph Grammars. In Graph-Theoretic Concepts in Computer Science, 20th International Workshop, WG '94, Herrsching, Germany, June 16-18, 1994, Proceedings, Ernst W. Mayr, Gunther Schmidt, and Gottfried Tinhofer (Eds.). 151-163. https://doi.org/10.1007/ 3-540-59071-4_45

[32] Nils Weidmann, Lars Fritsche, and Anthony Anjorin. 2020. A search-based and fault-tolerant approach to concurrent model synchronisation. In Proceedings of the 13th International Conference on Software Language Engineering, SLE 2020, Virtual Event, USA, November 16-17, 2020, Ralf Lämmel, Laurence Tratt, and Juan de Lara (Eds.). 56-71. https://doi.org/10.1145/3426425.3426932

[33] Bernhard Westfechtel. 2014. Merging of EMF models - Formal foundations. Softw. Syst. Model. 13, 2 (2014), 757-788. https://doi.org/10.1007/s10270-012-0279-3 